\title{
Bootstrapped Full-Swing CMOS Driver for Low Supply Voltage Operation
}

\author{
José C. García, Juan A. Montiel-Nelson \\ Institute for Applied Microelectronics, \\ Dept. of Electronic Eng. and Automation, \\ University of Las Palmas de Gran Canaria, \\ E-35017 Las Palmas de Gran Canaria, Spain. \\ \{jcgarcia, montiel\}@iuma.ulpgc.es
}

\begin{abstract}
This paper reports a high speed and low power consumption direct-indirect bootstrapped full-swing CMOS inverter driver circuit (bfi-driver). The simulation results, based on $0.13 \mu \mathrm{m}$ triple well CMOS technology, show that, when operated at $1 \mathrm{~V}$, bf-driver is $94 \%$ faster and consumes $22 \%$ less power compared to a counterpart direct bootstrap circuit [1].
\end{abstract}

\section{Introduction}

Driving large capacitive loads limits the performance of CMOS circuits at low voltages. Use of standard high speed BiCMOS drivers in not viable in low voltage portable electronic systems. In this paper, a combined direct and indirect bootstrapped inverter driver circuit in triple well CMOS technology is proposed and analyzed. The features of $b f-$ driver is compared to a counterpart direct bootstrapped fullswing circuit. The chosen counterpart circuit of Fig. 1 is a fully CMOS re-implementation of BiCMOS driver scheme in [1] where NMOS transistors M8 and M9 are the replacement for the original NPN bipolar transistors.

\subsection{Previous Work}

Full-swing BiCMOS/BiNMOS logic circuits using bootstrapping in the pull-up section for low supply voltage down to $1 \mathrm{~V}$ were proposed in [1] to outperform other driver circuits when fanout or the load capacitance is high. A bootstrapped circuit for CMOS and BiCMOS dynamic logic gates to enhance the speed performance was reported in [2]. The bootstrapping techniques proposed in [2] and [3] have been shown to give high performance at low supply voltages.

\author{
Saeid Nooshabadi \\ School of Electrical Engineering and Telecom. \\ University of New South Wales, \\ Sydney, NSW 2051 AU. \\ saeid@unsw.edu.au
}

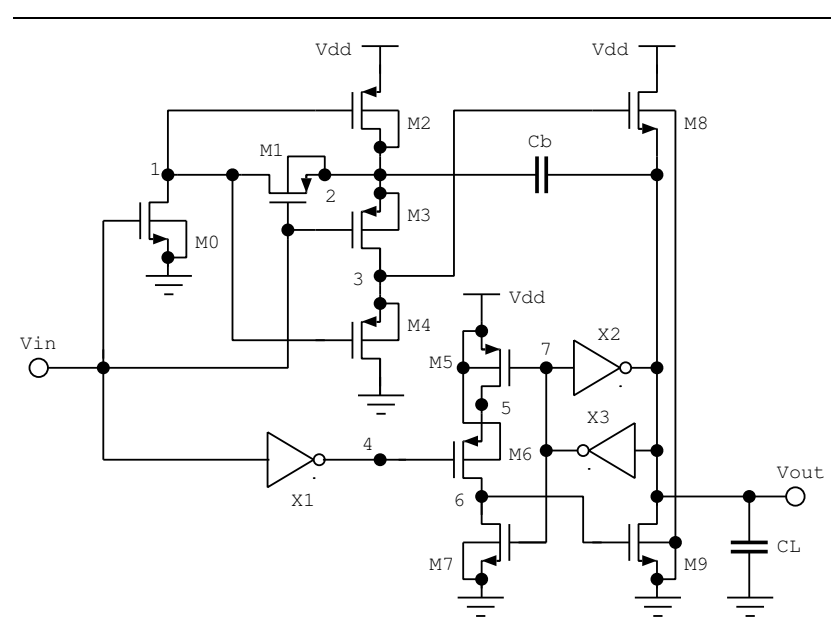

Figure 1. Circuit structure of bootstrapped CMOS in [1].

\section{Driver Circuit Structure}

Fig. 2 presents the circuit structure for bfi-driver. In this driver circuit, we use two bootstrap capacitors Cb1 and $\mathrm{Cb} 2$, and only one inverter X0. M0 and M3 are used to charge the bootstrap capacitors $\mathrm{Cb} 1$ and $\mathrm{Cb} 2$, respectively. M1 and M4 are the bootstrap transistors. M2 forms the pulldown network, while M5 forms the pull-up network. The parameters in Table 1 were used to design and simulate both driver circuits. $\mathrm{Cb}, \mathrm{Cb} 1$ and $\mathrm{Cb} 2$ are realized using NMOS transistors $\mathrm{MCb}, \mathrm{MCb} 1$ and $\mathrm{MCb} 2$, respectively, of Table 1. The channel length of all transistors is $0.13 \mu \mathrm{m}$.

\section{Circuit Operation}

In the proposed circuit structure of Fig. 2, when $\mathrm{Vin}$ is high, the node 1 is set to low via the inverter $\mathrm{X} 0$, and $\mathrm{M} 0$ is turned on. As a result, the bootstrapping capacitor $\mathrm{Cb} 1$ is charged to $\mathrm{Vdd}$ (node 2 at $\mathrm{Vdd}$ and node 1 at ground po- 


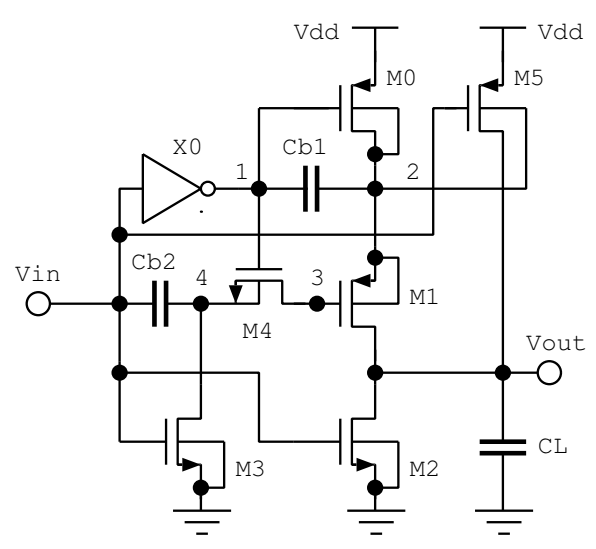

Figure 2. Schematic of the proposed bootstrapped full-swing CMOS inverter driver.

tential). M1, M4 and M5 are all turned off. M3 transistor is turned on causing $\mathrm{Cb} 2$ to charge (node $\mathrm{Vin}$ at $\mathrm{Vdd}$ and node 4 at ground potential). At the same time, M2 is driven by Vin and bfi-driver is pulled down, the output Vout is set to low. When Vin goes low, the node 1 is set to high, M0, M2, and M3 are turned off. M5 is turned on to pull-up the output. Simultaneously, M4 turns on allowing the gate of M1 to be strongly driven low due to the bootstrapping effect of $\mathrm{Cb} 2$. Meanwhile, the opposite bootstrapping effect of $\mathrm{Cb} 1$ directly couples to the output node Vout further assisting in the pull-up, and improving the switching speed.

\section{Comparative Evaluation}

Both driver circuits were implemented using UMC triple well $0.13 \mu \mathrm{m} 1.2 \mathrm{~V} / 3.3 \mathrm{~V}$ CMOS process. Active areas for the proposed circuit and the circuit in [1] are $35.86 \mu \mathrm{m}^{2}$ and $44.17 \mu \mathrm{m}^{2}$, respectively. The circuits were simulated at $500 \mathrm{MHz}$ input frequency with $100 \mathrm{ps}$ rise and fall times, at supply voltages of $1 \mathrm{~V}$ and $1.2 \mathrm{~V}$, and output capacitive load in the range of 10 to $100 \mathrm{fF}$. Simulation results show that at the power supply of $1 \mathrm{~V}$ our $b f$-driver is $94 \%$ faster (19ps delay at $100 \mathrm{fF}$ load), and its power consumption $(1.28 \mathrm{~mW})$ is $22 \%$ lower than the driver in [1]. Fig. 3 illustrates Figures of Merit (delay power product) versus load capacitance for two drivers. As seen the Figure of Merit for bfi-driver is 23.6-37.5 times smaller.

\section{Conclusions}

This paper presented a new high performance low power direct-indirect CMOS bootstrapped driver design (bfi-driver). Under a condition of $1 \mathrm{~V}$ voltage power supply and a capacitive loading of $100 \mathrm{fF}$, the de- lay and the power consumption associated with $b f$-driver are $19 \mathrm{ps}$ and $1.28 \mathrm{~mW}$, respectively.

\begin{tabular}{||c|c|c|c|c|c||}
\hline \hline \multicolumn{3}{|c|}{ Driver in [1] } & \multicolumn{3}{c||}{ bfi-driver } \\
\hline \hline Transistor(s) & Type & $\begin{array}{c}\text { Width } \\
(\mu m)\end{array}$ & Transistor(s) & Type & $\begin{array}{c}\text { Width } \\
(\mu m)\end{array}$ \\
\hline PX1, PX3, M1 & PMOS & 0.28 & PX0 & PMOS & 5 \\
\hline NX1 & NMOS & 5 & NX0 & NMOS & 0.28 \\
\hline PX2 & PMOS & 15 & M0 & PMOS & 14 \\
\hline NX2 & NMOS & 0.28 & M1 & PMOS & 10 \\
\hline NX3 & NMOS & 0.5 & M2 & NMOS & 19 \\
\hline M0, MCb & NMOS & 50 & M3 & NMOS & 0.28 \\
\hline M2 & PMOS & 1 & M4 & NMOS & 80 \\
\hline M3 & PMOS & 2 & MCb1 & NMOS & 60 \\
\hline M4 & PMOS & 0.28 & MCb2 & NMOS & 100 \\
\hline M5 & PMOS & 50 & - & - & - \\
\hline M6 & PMOS & 5 & - & - & - \\
\hline M7 & NMOS & 35 & - & - & - \\
\hline M8 & NMOS & 1 & - & - & - \\
\hline M9 & NMOS & 60 & - & - & - \\
\hline \hline
\end{tabular}

Triple well $0.13 \mu \mathrm{m} 1.2 \mathrm{~V} / 3.3 \mathrm{~V}$ process technology from UMC

Table 1. Channel widths for driver transistors.

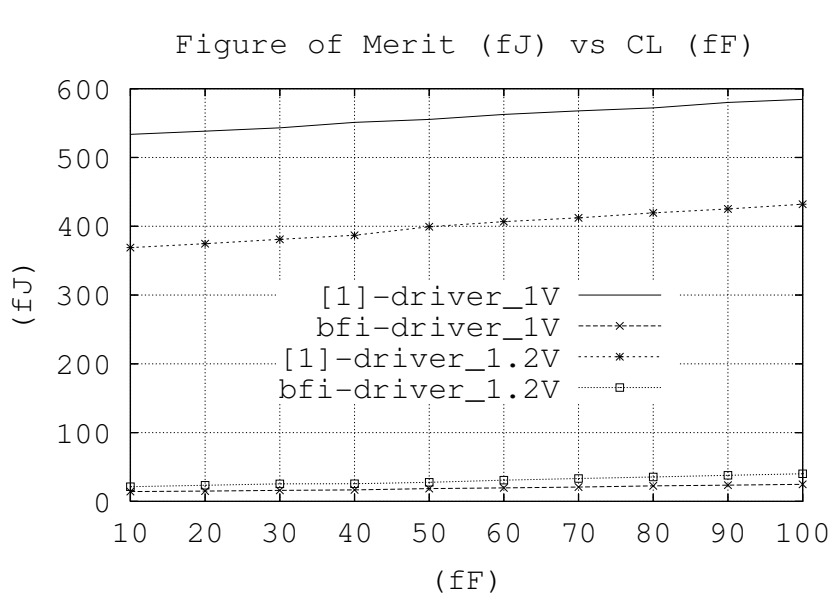

Figure 3. Delay $\times$ power versus loading.

\section{References}

[1] A. Bellaouar, M. I. Elmasry and S. H. K. Embabi, "Bootstrapped full-swing BiCMOS/BiNMOS logic circuits for 1.2$3.3 \mathrm{~V}$ supply voltage regime," IEEE J. Solid-State Circuits, vol. 30, no. 6, pp. 629-636, June 1995.

[2] J. H. Lou, and J. B. Kuo, "1.5V CMOS and BiCMOS Bootstrapped Dynamic Logic Circuits Suitable for Low-Voltage VLSI," VLSI Technology, Systems, and Aplications, pp. 279282, June 1997.

[3] J. C. García, J. A. Montiel-Nelson, J. Sosa and H. Navarro, "A direct bootstrapped CMOS large capacitive-load driver circuit," Design, Automation and Test in Europe Conference and Exhibition, vol. 1, pp. 680-681, Feb. 2004. 